\title{
Results on particle identification with silica aerogel
}

\section{Marta Calvi*}

Dipartimento di Fisica, Università di Milano Bicocca and INFN, Piazza della Scienza 3, IT-20126 Milan, Italy

E-mail: marta.calviemib.infn.iti

\section{T. Bellunato, C. Matteuzzi, M. Musy, P. Negri and M. Paganoni}

Dipartimento di Fisica, Università di Milano Bicocca and INFN, Milan, Italy

\section{A.R.Buzykaev, E.A.Kravchenko and A.P.Onuchin}

Budker Institute of Nuclear Physics, Novosibirsk, Russia

\section{A.F.Danilyuk}

Boreskov Institute of Catalysis, Novosibirsk, Russia

\section{S. Wotton}

University of Cambridge, UK

A. Braem, E. Chesi, C. Joram, D. Liko, N. Neufeld, J. Seguinot, D. Voillat, and P. Weilhammer

CERN, European Organization for Nuclear Research, Geneva, Switzerland

\section{T. Duane and S.Jolly}

Imperial College, London, UK

\begin{abstract}
Different samples of silica aerogel, which will be used as one of the radiators in the Ring Imaging Cherenkov of the LHCb experiment, were tested in a charged particle beam at CERN. Pions and protons with momenta ranging from 6 to $10 \mathrm{GeV} / \mathrm{c}$ traversed tiles of different thickness and different optical qualities, both hydrophobic and hydrophilic. Data were taken using as photodetectors up to four large diameter pad HPDs, produced at CERN. They showed an excellent signal to noise ratio, and provided coverage of up to $2 / 5$ of the ring. Results on photon yield and resolution on the Cherenkov angle are discussed. Separation between pions and protons is demonstrated over the whole momentum range.
\end{abstract}

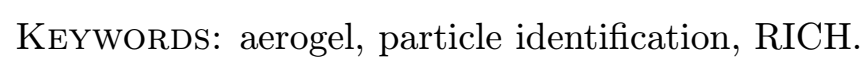




\section{Introduction}

$\mathrm{LHCb}$ is a single arm spectrometer which will take data at the Large Hadron Collider at CERN, in proton-proton collisions with center of mass energy of $14 \mathrm{TeV}$, starting in 2006 [i]. The main purpose of the experiment is to make CP violation measurements in the B sector. Particle identification is of fundamental importance for LHCb and hadrons have to be identified in a wide momentum spectrum, ranging from few $\mathrm{GeV} / \mathrm{c}$ up to about $150 \mathrm{GeV} / \mathrm{c}$. This is provided by two RICH detectors using three radiators with different refraction indices [ìi $]$.

RICH 1 contains both the aerogel with refractive index $\mathrm{n}=1.03$, and the $C_{4} F_{10}(\mathrm{n}=$ 1.014) radiators. Tilted spherical mirrors project the ring images of both radiators onto the photodetector planes. The Cherenkov light from the aerogel allows hadron identification in the region from 2 to $11 \mathrm{GeV} / \mathrm{c}$, while that from $C_{4} F_{10}$ covers the range from 10 to 50 $\mathrm{GeV} / \mathrm{c}$. RICH 2 contains $C F_{4}$ gas $(\mathrm{n}=1.005)$ which is well suited for the identification of the higher momentum particles.

\section{Aerogel characterization}

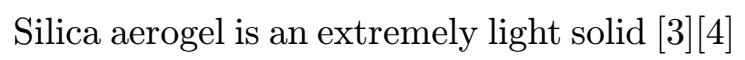
$\left(\right.$ density $\left.=0.1-0.2 \mathrm{~g} / \mathrm{cm}^{3}\right)$ with a netlike structure of a scale of few $\mathrm{nm}$ and a refraction index in the range 1.01-1.10. Part of the Cherenkov light produced by charged particles traversing aerogel undergoes Rayleigh scattering. The transmittance can be expressed as a function of the wavelength as:

$$
T=A \exp \left(-C d / \lambda^{4}\right)
$$

where $A$ represents the transmittance in the long wavelength region, $d$ is the aerogel thickness, in $\mathrm{cm}$, and $C$ is called the clarity coefficient. Small values of clarity are crucial in order to use aerogel in a RICH detector [יin].

Two qualities of aerogel have been tested in a beam: the first one was produced by Matsushita Electric, Ltd, (Japan), it is of SP30 type,

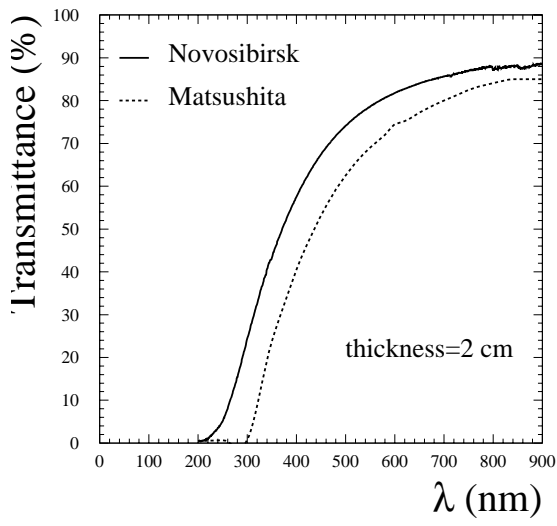

Figure 1: The transmittance as a function of wavelength for two aerogel samples $2 \mathrm{~cm}$ thick, one of hydrofillic type produced in Novosibirsk (solid line), and one of hydrophobic type (dotted line) produced by Matsushita. hydrophobic. Tiles of different dimensions were used: $5.5 \times 5.5 \times 1 \mathrm{~cm}^{3}$ and $8 \times 8 \times 2 \mathrm{~cm}^{3}$. The second sample of aerogel tiles was produced by the Boreskov Institute of Cathalisis, Novosibirsk (Russia) [i $\left[\begin{array}{l}4 \\ 1\end{array}\right]$, with dimensions $10 \times 10 \times 2 \mathrm{~cm}^{3}, 7 \times 8 \times 4 \mathrm{~cm}^{3}$ and $10 \times 10$ $\times 4 \mathrm{~cm}^{3}$. This aerogel is hydrofillic and for this reason nitrogen was flushed through the vessel during the test, in order to protect the aerogel against humidity.

\footnotetext{
${ }^{*}$ Speaker.
} 
The optical properties of the aerogel samples have been tested in advance, by measuring with a spectrophotometer, the light transmission as a function of the wavelength and fitting with equation $2 \overline{2} .1$ to determine the $C$ coefficient. In Figure 1, for example, clarities $C=96 \times 10^{-4} \mu m^{4}$ $\mathrm{cm}^{-1}$ and $C=56 \times 10^{-4} \mu \mathrm{m}^{4} \mathrm{~cm}^{-1}$ were measured for one sample of the Matsushita and the Novosibirsk aerogel, respectively. Ageing properties of the aerogel have also been studied. The radiation hardness of the aerogel has been monitored exposing a sample to a gamma ray source ${ }^{60} \mathrm{Co}$ with an intensity of about 4 Gy per minute. As it is shown in Figure 2 , no significant degradation of the transmission properties have been found, up to a dose of more than 20 MRad.

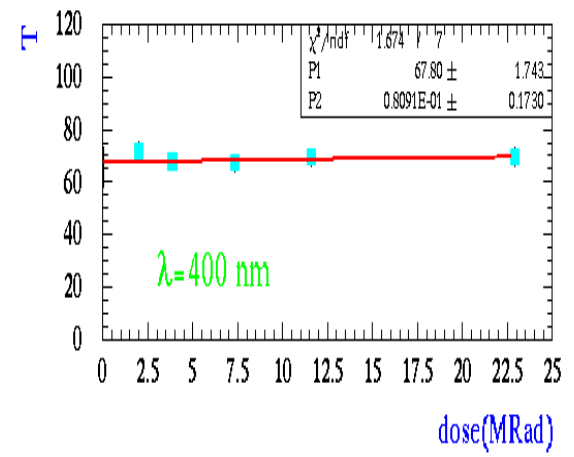

Figure 2: The transmittance as a function of the absorbed dose. The aerogel is $1 \mathrm{~cm}$ thick, Novosibirsk type. Analogous measurements are planned after strong irradiation with charged particles.

\section{Test beam setup}

Two tests have been performed with silica aerogel as RICH radiator in November 1999 and May 2001, respectively. They used the same experimental setup consisting of a light tight aluminum vessel, painted black, about $1 \mathrm{~m}$ long, containing the aerogel radiator which is traversed by the beam. Different thickness $(2$ to $8 \mathrm{~cm}$ ) of aerogel were exposed, using 1 to 6 tiles stacked together.

Cherenkov light produced in the aerogel is reflected by a spherical mirror of $1.2 \mathrm{~m}$ radius of curvature, towards the detector plane. Two photodetectors were mounted during the first test beam while four photodetectors were used in the second one. For $\mathrm{n}=1.03$, charged particles with $\beta \simeq 1$ are expected to produce

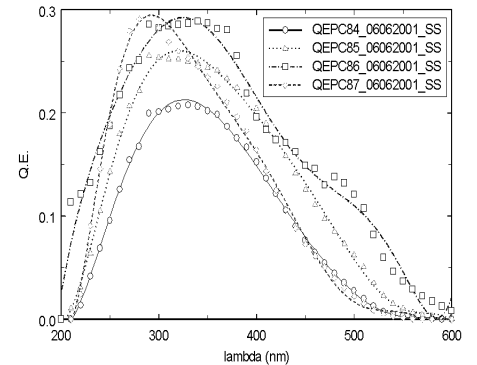

Figure 3: Quantum efficiency of the four pad-HPDs used in the 2001 test beam.

a Cherenkov light cone of $242 \mathrm{mrad}$. About 1/10 of the resulting ring was covered by each photodetector.

The vessel was installed in the East Hall experimental area at CERN, in the T7 secondary beamline of the Proton Syncrotron, with momenta up to $10 \mathrm{GeV} / \mathrm{c}$. The polarity and momentum of the secondary beam can be selected, with a momentum bite of $1 \%$. The negative polarity gives a pure $\pi^{-}$beam, while the positive polarity yields a mixture of $\pi^{+}$ and protons. In order to determine the direction and position of the incoming particles, two silicon planes were located upstream the vessel, and one downstream, with $1.3 \mathrm{~mm} \times$ $1.3 \mathrm{~mm}$ pixels. The measured beam divergence was about $1 \mathrm{mrad}$. 
The photodetectors used in this test are padHPDs, developed and produced at CERN [6] 60 . The pad-HPD is a fast, large area $(12.7 \mathrm{~cm}$ diameter), highly sensitive Hybrid Photodiode with pixelized readout with 2048 channels. The fountain shaped electric field, gives a 2.4 fold demagnified image. The quantum efficiency of the bialkali photocatodes is shown in Fig. 'i3!r The low noise analogue chain was based on Viking VA2 chips. During the test beam the tubes were operated at a voltage ranging from 14 to $16 \mathrm{KV}$. The average noise per pixel was lower than 1 per mille. Some pixels showing higher noise were masked for the off-line analysis.

\section{Results}

In Fig. ${ }_{14}^{4}$ the display of the four pad-HPD is shown, where the Cherenkov ring produced by a $9 \mathrm{GeV} / \mathrm{c}$ pion beam is clearly visible.

The number of produced photoelectron has been measured with 1999 data, from the radial distribution of accumulated events. A ring was fitted across the arcs measured in the pad-HPDs with a resolution in the radius of about $3 \mathrm{~mm}$. A ring region was defined as $\pm 9 \mathrm{~mm}$ around the fitted value of the radius, and was used to evaluate the photoelectron yield on the ring from the aerogel. A cut at ADC charge $>15$ was applied and the noise contribution was subtracted. The measured yield is extrapolated to the full ring taking into account geometrical acceptance and signal loss corrections. In Fig. $\underline{5}_{1}^{\prime}$ data are compared with the expectations from a Monte Carlo

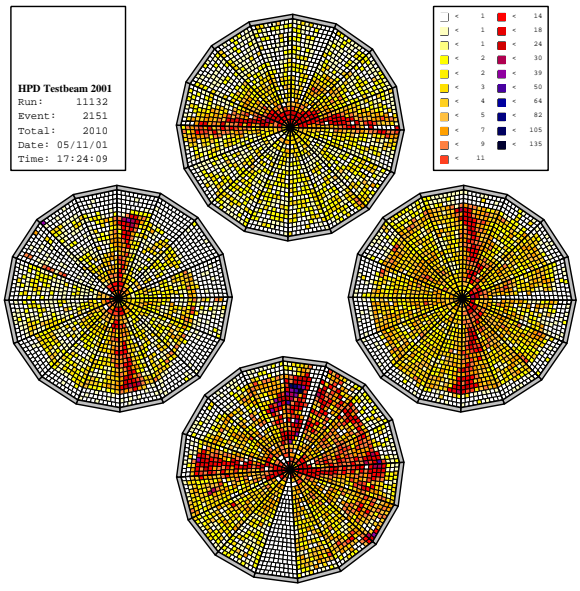

Figure 4: On-line display of four padHPD measuring the Cherenkov ring produced in the aerogel by a $9 \mathrm{GeV} / \mathrm{c}$ pion beam. The distances between the HPDs have been reduced for the figure. simulation which uses the measured clarity. The estimation for photoelectrons outside the ring, which are mainly due to Rayleigh scattering, is also shown. Fig. $\overline{6}_{1}^{7}$ shows the radius distributions from two different rings produced by pions and protons present in the beam. The percentage of protons in the beam was increasing with momentum from about $50 \%$ at $6 \mathrm{GeV} / \mathrm{c}$ to $70 \%$ at $10 \mathrm{GeV} / \mathrm{c}$.

The Cherenkov angle was directly determined using an extrapolation procedure applied to each photon after fixing the charged particle direction. Results for a positive pion and proton beam at $8 \mathrm{GeV} / \mathrm{c}$ are shown in Fig. ini The angular resolution in not yet optimal. The main contribution to the resolution comes from achromaticity of the emitted photons. Data were taken also with a filter interposed at the exit side of the aerogel stack, in order to 

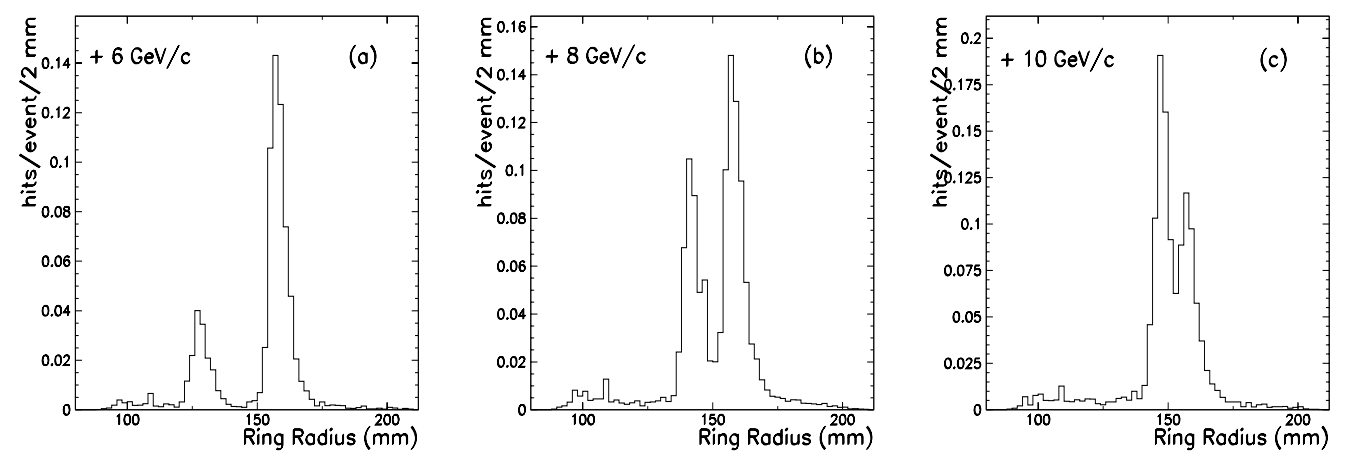

Figure 6: Number of pixels hit per event as a function of the ring radius, for different beam momenta.

absorb high energy photons, which are the most affected by Rayleigh scattering. Different filters were tried and an optimization in under study.

\section{Conclusions}

Results obtained by exposing samples of silica aerogel to pion and proton beams with momenta between 6 and $10 \mathrm{GeV} / \mathrm{c}$ have been presented.

Separate Cherenkov rings produced by the different particles were reconstructed obtaining pion/proton separation on the whole momentum range. The number of photoelectrons was measured as a function of aerogel thickness and was found in agreement with Monte Carlo expectations.

\section{Acknowledgments}

We acknowledge the support of INTAS through the contract INTAS-679. We would like to thank R.Mazza

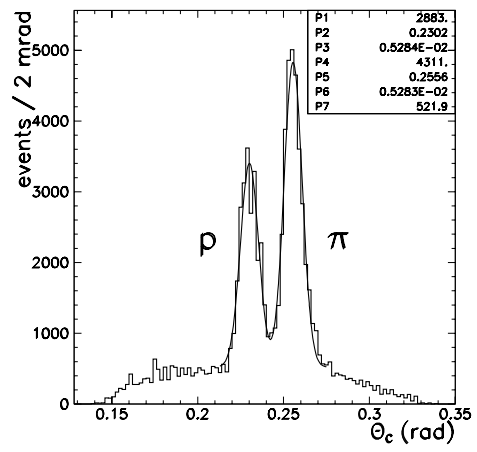

Figure 7: Cherenkov angle reconstructed for pions and protons in a 8 $\mathrm{GeV} / \mathrm{c}$ beam. for his support with the setup construction.

\section{References}

[1] S. Amato et al., Cern, Geneva, Tech. Rep. CERN/LHCC 98-4, Feb. 1998.

[2] S. Amato et al., Cern, Geneva, Tech. Rep. CERN/ LHCC/2000-0037, Sep. 2000.

[3] H.Yokogawa, M.Yokoyama J. Non-Cryst. Solids 186 (1995) 23.

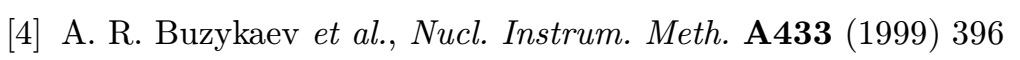

[5] R. De Leo et al., iNucl. Instrum. Meth. A401 19997 187'

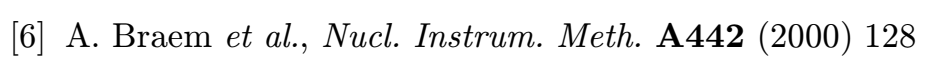

